\title{
Effect of Seed Treatments and Storage Containers on Viability and Vigour of Rice (Oryza sativa L.) Variety ADT (R) 46 Seeds
}

\author{
K. Raja ${ }^{1^{*}}$ and K. Sasikala ${ }^{2}$ \\ ${ }^{1}$ Department of Seed Science and Technology, Seed Centre, Tamil Nadu Agricultural \\ University, Coimbatore - 641 003, Tamil Nadu, India \\ ${ }^{2}$ Agricultural College \& Research Institute, Tamil Nadu Agricultural University, \\ Eachangkottai, Thanjavur - 614 902, Tamil Nadu, India
}

*Corresponding author

\section{A B S T R A C T}

\section{Keywords \\ Rice, Seed storage, Viability, Vigour, anti- oxidant, $\alpha$ - tocopherol, Vitamin - E \\ Article Info \\ Accepted: \\ 20 August 2018 Available Online: 10 September 2018}

\begin{abstract}
Rice variety ADT (R) 46 seeds have the problem of poor storage and therefore, to increase the storability of the seeds an experiment was conducted at Tamil Nadu Rice Research Institute, Aduthurai during 2016-18 by treating the seeds with different chemicals and leaf extracts. The results showed that the seeds treated with antioxidant, $\alpha$ - tocopherol (Vitamin - E) @ $1 \%$ for $18 \mathrm{~h}$ and stored in polylined gunny bag have recorded the higher germination $(95 \%)$ and seedling vigour upto six months compared to control. However, germination and vigour were reduced significantly after six months irrespective of the treatments. The varietal genetic makeup and climatic factor are the most probable causes for its poor storage.
\end{abstract}

\section{Introduction}

Rice (Oryza sativa L.) is an important stable food crop for about $60 \%$ of the world's population. The production and supply of quality seed in rice plays a vital role to meet the growing population. It has been shown experimentally that only by using good quality healthy seed, rice yield could be increased by 10-15\% (Akter et al., 2015). In rice, there is no much problem in seed storage if the crop is well maintained during production period. Paderes et al., (1997) reported that the germination of rice varieties in storage does not suffer much as compared to other cereals.
However, rice seed stored for long-term is invariably exposed to different climatic adversities like extreme summer, winter and monsoons etc. requires a great deal of effort to safeguard it.

Therefore, seed storage is an important aspect of any sound seed program, because badly stored seeds are not much helpful to yield healthy and vigorous plant. A good quality seed may also be seriously deteriorated if stored under sub optimal condition. Considerable amount of works have been done on storage of rice seeds in relation to varied storage conditions in different parts of the 
world (Purushattam et al., 1996; Khalid et al., 2001). However, the seed viability and vigour largely depends on the genotypes, production and post-harvest conditions. During the storage, several aspects have a direct effect on the seeds feasibility, such as seed water content during storage, conservation packages, temperature and relative humidity of the air and the storage environment, gas exchange and characteristics of the seed skin (Caldwell et al., 2005; Toledo et al., 2009). Marques et al., (2014) found that the rice seeds that stored in natural environment without controlling temperature and relative humidity showed lower physiological quality. The tropical climate with high temperature and high relative humidity along with unscientific storage conditions adversely affect the preservation of cereal grains, oilseeds, etc., which lead to the total loss of seed quality (Begum et al., 2013).

It is incontestable that proper seed treatment and storage methods can substantially improve the quality of seed and significantly increase the yield. Sultana et al., (2016) found zero germination in rice seeds stored in earthen, tin and plastic containers after six months of storage with minimum germination in gunny bag. The antioxidant substances like ascorbic acid, tocopherol and glutathione had a positive effect on the seedling vigour (Basra et al., 2006; Draganic and Lekic, 2012). Similarly, seed treatment with biofungicide has been found as superior in increasing germination and vigour in rice seeds (Akter and Hossain, 2015). Therefore, an experiment was conducted in the rice variety ADT (R) 46 seeds by treating with different antioxidants as to manage its poor storage behavior.

\section{Materials and Methods}

The storage experiment in rice variety ADT (R) 46 seeds was conducted at Tamil Nadu Rice Research Institute, Aduthurai (India) for two years during 2016 and 2017. Usually, ADT (R) 46 rice variety is raised during Thaladi season (September - October) and harvested during January - February in Cauvery Delta region of Tamil Nadu.

Accordingly, the present experimental seed material was harvested during February and used for conducting the storage experiment. The freshly harvested seeds were given the following pre-storage seed treatments viz., $\mathrm{T}_{1}$ Untreated control, $\mathrm{T}_{2}$ - Seed soaking in water for $18 \mathrm{~h}, \mathrm{~T}_{3}-$ Seed fortification in $\mathrm{KH}_{2} \mathrm{PO}_{4} @$ $1 \%$ for $18 \mathrm{~h}, \mathrm{~T}_{4}$ - Seed fortification in Prosopis juliflora leaf extract @ $1 \%$ for 18 h, $\mathrm{T}_{5}$ - Seed fortification in $\alpha$ - tocopherol (Vitamin - E) @ $1 \%$ for 18 h, $\mathrm{T}_{6}$ - Seed fortification in ascorbic acid (Vitamin - C) @ $1 \%$ for $18 \mathrm{~h}, \mathrm{~T}_{7}$ - Seed treatment with halogen mixture $\left(\mathrm{CaOCl}_{2}+\mathrm{CaCO}_{3}+\right.$ Albezia amara @ 5:4:1 ratio)@ $3 \mathrm{~g} \mathrm{~kg}^{-1}$ and $\mathrm{T}_{8}$ - Seed treatment with halopolymers@ $3 \mathrm{ml} \mathrm{kg}{ }^{-1}$ of seeds.

Then, the treated seeds were packed in the storage containers viz., gunny bag $\left(\mathrm{C}_{1}\right)$ and polylined gunny bag $\left(\mathrm{C}_{2}\right)$ and stored under ambient condition. The seed samples were drawn at bimonthly intervals for the evaluation of moisture content, germination and seedling vigour.

The germination test was conducted in four replications by placing the 100 seeds in each replication and evaluated at $14^{\text {th }}$ day of test (ISTA, 1999). The seedling vigour index was computed by multiplying the germination percentage with seedling length (Abdul-Baki and Anderson, 1973). The data collected in two years were pooled and analyzed (Panse and Sukhatme, 1967) at $5 \%$ probability level.

\section{Results and Discussion}

The pooled analysis results indicated that the significant increase in seed moisture content was recorded in both gunny bag and polylined 
gunny bag during the 10 months storage. However, the rate of increase was lesser in polylined gunny bag than the gunny bag. In this regard, the initial moisture content of 11.1 and 10.1 per cent was increased to 12.0 and 11.0 at 10 months storage in gunny bag and polylined gunny bag (Table 1). Regarding the seed viability, initial seed germination $(97 \%)$ was reduced drastically (39\%) during 10 months storage irrespective of treatments and containers. Among the containers, polylined gunny bag have performed better in maintaining the germination. In which, the seed fortification in $\alpha$-tocopherol @ $1 \%$ for $18 \mathrm{~h}$ and stored in polylined gunny bag has recorded higher germination $(95 \%)$ upto six months storage when compared to control (80 $\%)$. However, the germination of this treatment was declined severely and recorded 52 per cent at eight months storage. Other treatments were also showed similar trend of rapid decline in germination. In this regard, viability and vigour of the seeds decreased slowly upto six months and thereafter, drastic decline was recorded irrespective of the seed treatments (Table 2). Similar findings of germination and vigour reduction in rice seed during storage were recorded by many workers (Biradar Patil and Shekhargouda, 2007; Marques et al., 2014; Sultana et al., 2016; Anil Sebastian and Selvaraju, 2017).

Moisture difference due to relative humidity might have resulted in wetting of paddy seed (Kad et al., 2016) and it can be minimized by storing the seeds in proper containers like gunny bag (Sultana et al., 2016), polythene bag (Pameri et al., 2016) or vacuum polythene bag (Jyoti, 2017). Kameswara Rao and Jackson (1996) found the significant differences among the cultivar in seed storage longevity and therefore, it is advisable during storage to monitor more frequently the viability of accessions with intrinsically poor storage characteristics. This variation in storability among the genotypes was obviously due to the genetic control to resist deterioration during storage (Yogalakshmi et al., 1996). Marques et al., (2014) found the seeds of cultivar Seleta, regardless of the environment, maintained germination above the minimum required for commercialization until six months of storage.

The decline in viability percentage may be attributed to ageing effect leading to depletion of food reserves and decline in synthetic activity of embryo. The free radical formation contributes much in the ageing of the seeds and it can be minimized by seed treatment with the antioxidant substances like ascorbic acid, tocopherol and glutathione (Draganic and Lekic, 2012). Basra et al., (2006) found that the aqueous solution of ascorbic acid improved the germination and seedling vigour in rice during seed storage.

Similar findings were also observed in the present study in which the anti-oxidant, $\alpha$ tocopherol have maintained the germination and seedling vigour upto six months. However, the poor storability of the rice variety ADT (R) 46 seeds is presumably due to the genetic makeup of the variety.

While observing the environmental factors, the higher relative humidity (88 and $90.2 \%$ ) was recorded during the seed storage period in both the years at Aduthurai region (Table 6). Also, the humidity was more than 80 per cent in all the months except few cases.

The rainfall during North East Monsoon season particularly during September to December was higher which ultimately increased the relative humidity to more than 90 per cent. This higher relative humidity might have contributed faster deterioration of the seeds even though it is treated with antioxidants. 
Table.1 Effect of seed treatment and storage container on moisture content (\%) in rice variety ADT (R) 46 (pooled data)

\begin{tabular}{|c|c|c|c|c|c|c|c|c|c|c|c|c|c|c|c|c|c|c|c|}
\hline \multirow{2}{*}{$\begin{array}{l}\text { Treat } \\
\text { ments }\end{array}$} & \multicolumn{3}{|c|}{ Initial } & \multicolumn{3}{|c|}{2 MAS } & \multicolumn{3}{|c|}{4 MAS } & \multicolumn{3}{|c|}{6 MAS } & \multicolumn{3}{|c|}{8 MAS } & \multicolumn{3}{|c|}{10 MAS } & \multirow[t]{2}{*}{ Mean } \\
\hline & $\mathbf{C}_{1}$ & $\mathrm{C}_{2}$ & $\mathbf{M}$ & $\mathrm{C}_{1}$ & $\mathrm{C}_{2}$ & $\mathbf{M}$ & $\mathrm{C}_{1}$ & $\mathrm{C}_{2}$ & $\mathbf{M}$ & $\mathbf{C}_{1}$ & $\mathrm{C}_{2}$ & $\mathbf{M}$ & $\mathbf{C}_{1}$ & $\mathrm{C}_{2}$ & $\mathbf{M}$ & $\mathrm{C}_{1}$ & $\mathrm{C}_{2}$ & $\mathbf{M}$ & \\
\hline$T_{1}$ & 11.3 & 10.1 & 10.7 & 11.4 & 10.1 & 10.8 & 11.3 & 10.2 & 10.7 & 12.2 & 11.2 & 11.7 & 12.5 & 11.5 & 12.0 & 12.1 & 11.1 & 11.6 & 11.2 \\
\hline$T_{2}$ & 10.9 & 10.2 & 10.6 & 11.1 & 10.3 & 10.7 & 11.3 & 10.3 & 10.8 & 12.1 & 11.4 & 11.7 & 12.5 & 11.4 & 11.9 & 12.1 & 11.0 & 11.5 & 11.2 \\
\hline $\mathbf{T}_{\mathbf{3}}$ & 11.1 & 10.2 & 10.6 & 11.1 & 10.2 & 10.6 & 11.4 & 10.1 & 10.7 & 12.5 & 11.2 & 11.8 & 12.6 & 11.7 & 12.1 & 12.4 & 11.0 & 11.7 & 11.3 \\
\hline $\mathbf{T}_{4}$ & 11.1 & 10.0 & 10.5 & 11.2 & 10.1 & 10.6 & 11.4 & 10.2 & 10.8 & 12.5 & 11.3 & 11.9 & 13.1 & 11.5 & 12.3 & 12.0 & 11.0 & 11.5 & 11.3 \\
\hline$T_{5}$ & 11.2 & 10.2 & 10.7 & 11.1 & 10.3 & 10.7 & 11.6 & 10.7 & 11.2 & 12.7 & 11.6 & 12.1 & 12.9 & 12.0 & 12.4 & 11.4 & 11.6 & 11.5 & 11.4 \\
\hline $\mathrm{T}_{6}$ & 11.2 & 10.0 & 10.6 & 11.2 & 10.1 & 10.6 & 11.2 & 10.4 & 10.8 & 12.8 & 11.5 & 12.1 & 12.6 & 11.7 & 12.2 & 11.9 & 10.5 & 11.2 & 11.2 \\
\hline $\mathbf{T}_{7}$ & 11.1 & 10.1 & 10.6 & 11.1 & 10.2 & 10.6 & 11.0 & 10.5 & 10.8 & 12.5 & 11.5 & 12.0 & 13.2 & 12.0 & 12.6 & 11.9 & 11.1 & 11.5 & 11.3 \\
\hline $\mathbf{T}_{8}$ & 11.0 & 10.1 & 10.5 & 11.2 & 10.2 & 10.7 & 11.1 & 10.3 & 10.7 & 12.9 & 12.1 & 12.5 & 13.1 & 11.9 & 12.5 & 12.4 & 10.7 & 11.6 & 11.4 \\
\hline Mean & 11.1 & 10.1 & 10.6 & 11.1 & 10.2 & 10.6 & 11.3 & 10.3 & 10.8 & 12.5 & 11.5 & 12.0 & 12.8 & 11.7 & 12.2 & 12.0 & 11.0 & 11.5 & 11.3 \\
\hline & & $\mathrm{P}$ & $\mathrm{C}$ & $\mathrm{T}$ & $\mathrm{PC}$ & $\mathrm{CT}$ & PT & PCT & & & & & & & & & & & \\
\hline SEd & & 0.3 & 0.1 & 0.3 & 0.4 & 0.5 & 0.8 & 1.2 & & & & & & & & & & & \\
\hline $\mathrm{CD}(\mathrm{P}=$ & .05) & 0.6 & 0.3 & NS & NS & NS & NS & NS & & & & & & & & & & & \\
\hline
\end{tabular}

(MAS - Months after storage; M - Mean; P - Storage period; C - Container; T - Treatment) 
Table.2 Effect of seed treatment and storage container on germination (\%) in rice variety ADT (R) 46 (pooled data)

\begin{tabular}{|c|c|c|c|c|c|c|c|c|c|c|c|c|c|c|c|c|c|c|c|}
\hline \multirow{2}{*}{$\begin{array}{c}\text { Treat } \\
\text { ment } \\
\text { s }\end{array}$} & \multicolumn{3}{|c|}{ Initial } & \multicolumn{3}{|c|}{$2 \mathrm{MAS}$} & \multicolumn{3}{|c|}{$4 \mathrm{MAS}$} & \multicolumn{3}{|c|}{$6 \mathrm{MAS}$} & \multicolumn{3}{|c|}{$8 \mathrm{MAS}$} & \multicolumn{3}{|c|}{10 MAS } & \multirow[t]{2}{*}{ Mean } \\
\hline & $\mathbf{C}_{1}$ & $\mathrm{C}_{2}$ & $\mathbf{M}$ & $\mathbf{C}_{1}$ & $\mathbf{C}_{2}$ & $\mathbf{M}$ & $\mathbf{C}_{1}$ & $\mathrm{C}_{2}$ & $\mathbf{M}$ & $\mathrm{C}_{1}$ & $\mathrm{C}_{2}$ & $\mathbf{M}$ & $\mathbf{C}_{1}$ & $\mathrm{C}_{2}$ & $\mathbf{M}$ & $\mathbf{C}_{1}$ & $\mathrm{C}_{2}$ & $\mathbf{M}$ & \\
\hline$\overline{T_{1}}$ & 95 & 96 & 95 & 88 & 91 & 89 & 84 & 86 & 85 & 78 & 80 & 79 & 39 & 42 & 40 & 33 & 35 & 34 & 70 \\
\hline$\overline{T_{2}}$ & 97 & 98 & 97 & 94 & 96 & 95 & 88 & 87 & 87 & 81 & 79 & 80 & 43 & 45 & 44 & 35 & 37 & 36 & 73 \\
\hline $\mathbf{T}_{3}$ & 96 & 97 & 97 & 91 & 94 & 92 & 85 & 85 & 85 & 75 & 80 & 77 & 41 & 43 & 42 & 36 & 36 & 36 & 71 \\
\hline $\mathbf{T}_{4}$ & 95 & 95 & 95 & 90 & 93 & 91 & 80 & 90 & 85 & 76 & 69 & 72 & 47 & 46 & 46 & 37 & 38 & 37 & 71 \\
\hline$\overline{T_{5}}$ & 98 & 99 & 99 & 96 & 98 & 97 & 93 & 97 & 95 & 89 & 95 & 92 & 50 & 52 & 51 & 40 & 41 & 41 & 79 \\
\hline $\mathrm{T}_{6}$ & 95 & 96 & 96 & 90 & 90 & 90 & 80 & 84 & 82 & 74 & 74 & 74 & 40 & 43 & 41 & 35 & 35 & 35 & 69 \\
\hline $\mathbf{T}_{7}$ & 96 & 96 & 96 & 91 & 93 & 92 & 90 & 92 & 91 & 85 & 86 & 85 & 43 & 45 & 44 & 36 & 36 & 36 & 74 \\
\hline $\mathrm{T}_{8}$ & 98 & 98 & 98 & 96 & 97 & 96 & 88 & 94 & 91 & 76 & 85 & 80 & 69 & 63 & 66 & 59 & 56 & 57 & 81 \\
\hline \multirow[t]{2}{*}{ Mean } & 96 & 97 & 97 & 92 & 94 & 93 & 86 & 89 & 88 & 79 & 81 & 80 & 46 & 47 & 47 & 39 & 39 & 39 & 74 \\
\hline & & $\mathrm{P}$ & $\mathrm{C}$ & $\mathrm{T}$ & $\mathrm{PC}$ & $\mathrm{CT}$ & PT & PCT & & & & & & & & & & & \\
\hline SEd & & 0.1 & 0.09 & 0.2 & 0.2 & 0.2 & 0.4 & 0.6 & & & & & & & & & & & \\
\hline $\mathrm{CD}(\mathrm{P}$ & D5) & 0.3 & 0.19 & 0.4 & 0.4 & 0.5 & 0.9 & 1.3 & & & & & & & & & & & \\
\hline
\end{tabular}

(MAS - Months after storage; M - Mean; P - Storage period; C - Container; T - Treatment) 
Table.3 Effect of seed treatment and storage container on shoot length $(\mathrm{cm})$ in rice variety ADT (R) 46 (pooled data)

\begin{tabular}{|c|c|c|c|c|c|c|c|c|c|c|c|c|c|c|c|c|c|c|c|}
\hline \multirow{2}{*}{$\begin{array}{c}\text { Treat } \\
\text { ment } \\
\text { s }\end{array}$} & \multicolumn{3}{|c|}{ Initial } & \multicolumn{3}{|c|}{2 MAS } & \multicolumn{3}{|c|}{4 MAS } & \multicolumn{3}{|c|}{6 MAS } & \multicolumn{3}{|c|}{8 MAS } & \multicolumn{3}{|c|}{10 MAS } & \multirow[t]{2}{*}{ Mean } \\
\hline & $\mathrm{C}_{1}$ & $\mathrm{C}_{2}$ & $\mathbf{M}$ & $\mathrm{C}_{1}$ & $\mathrm{C}_{2}$ & $\mathbf{M}$ & $\mathrm{C}_{1}$ & $\mathrm{C}_{2}$ & $\mathbf{M}$ & $\mathrm{C}_{1}$ & $\mathrm{C}_{2}$ & $\mathbf{M}$ & $\mathrm{C}_{1}$ & $\mathrm{C}_{2}$ & $\mathbf{M}$ & $\mathbf{C}_{1}$ & $\mathrm{C}_{2}$ & $\mathbf{M}$ & \\
\hline $\mathbf{T}_{1}$ & 17.6 & 18.0 & 17.8 & 16.9 & 16.6 & 16.7 & 16.8 & 14.0 & 15.4 & 16.1 & 14.5 & 15.3 & 8.0 & 8.2 & 8.1 & 7.7 & 8.0 & 7.9 & 13.5 \\
\hline$\overline{T_{2}}$ & 16.9 & 17.0 & 17.0 & 16.0 & 16.8 & 16.4 & 17.1 & 15.8 & 16.4 & 16.9 & 15.7 & 16.3 & 9.1 & 9.3 & 9.2 & 8.5 & 8.8 & 8.7 & 14.0 \\
\hline $\mathbf{T}_{3}$ & 16.6 & 17.0 & 16.8 & 17.4 & 17.3 & 17.3 & 17.6 & 17.5 & 17.5 & 17.7 & 17.4 & 17.6 & 9.1 & 9.3 & 9.2 & 8.5 & 8.7 & 8.6 & 14.5 \\
\hline $\mathbf{T}_{4}$ & 17.9 & 18.1 & 18.0 & 16.3 & 16.8 & 16.5 & 18.4 & 16.4 & 17.4 & 17.8 & 18.5 & 18.1 & 15.8 & 9.4 & 12.6 & 9.0 & 9.1 & 9.1 & 15.3 \\
\hline$T_{5}$ & 18.4 & 18.0 & 18.2 & 16.5 & 16.9 & 16.7 & 18.4 & 17.7 & 18.0 & 18.0 & 17.8 & 17.9 & 15.3 & 14.5 & 14.9 & 11.1 & 12.8 & 11.9 & 16.3 \\
\hline $\mathrm{T}_{6}$ & 17.0 & 17.8 & 17.4 & 16.0 & 15.2 & 15.6 & 16.9 & 16.2 & 16.6 & 16.6 & 15.9 & 16.3 & 8.5 & 9.1 & 8.8 & 8.4 & 8.5 & 8.5 & 13.8 \\
\hline $\mathrm{T}_{7}$ & 17.7 & 17.8 & 17.7 & 16.6 & 16.7 & 16.6 & 15.9 & 17.7 & 16.8 & 15.3 & 16.1 & 15.7 & 9.1 & 9.4 & 9.2 & 8.5 & 8.8 & 8.6 & 14.1 \\
\hline $\mathrm{T}_{8}$ & 17.1 & 17.2 & 17.1 & 16.2 & 16.3 & 16.2 & 15.5 & 13.5 & 14.5 & 15.5 & 16.8 & 16.1 & 13.4 & 15.3 & 14.4 & 8.6 & 11.0 & 9.8 & 14.7 \\
\hline Mean & 17.4 & 17.6 & 17.5 & 16.5 & 16.5 & 16.5 & 17.1 & 16.1 & 16.6 & 16.7 & 16.6 & 16.6 & 11.0 & 10.5 & 10.8 & 8.8 & 9.5 & 9.1 & 14.5 \\
\hline & & $\mathrm{P}$ & $\mathrm{C}$ & $\mathrm{T}$ & PC & $\mathrm{CT}$ & PT & PCT & & & & & & & & & & & \\
\hline SEd & & 0.02 & 0.01 & 0.02 & 0.03 & 0.03 & 0.06 & 0.08 & & & & & & & & & & & \\
\hline CD (P & $0.05)$ & 0.04 & 0.02 & 0.05 & 0.06 & 0.07 & 0.12 & 0.16 & & & & & & & & & & & \\
\hline
\end{tabular}

(MAS - Months after storage; M - Mean; P - Storage period; C - Container; T - Treatment) 
Table.4 Effect of seed treatment and storage container on root length $(\mathrm{cm})$ in rice variety ADT (R) 46 (pooled data)

\begin{tabular}{|c|c|c|c|c|c|c|c|c|c|c|c|c|c|c|c|c|c|c|c|}
\hline \multirow{2}{*}{$\begin{array}{c}\text { Treat } \\
\text { ment } \\
\text { s }\end{array}$} & \multicolumn{3}{|c|}{ Initial } & \multicolumn{3}{|c|}{2 MAS } & \multicolumn{3}{|c|}{$4 \mathrm{MAS}$} & \multicolumn{3}{|c|}{6 MAS } & \multicolumn{3}{|c|}{8 MAS } & \multicolumn{3}{|c|}{10 MAS } & \multirow[t]{2}{*}{ Mean } \\
\hline & $\mathbf{C}_{1}$ & $\mathrm{C}_{2}$ & $\mathbf{M}$ & $\mathrm{C}_{1}$ & $\mathrm{C}_{2}$ & $\mathbf{M}$ & $\mathbf{C}_{1}$ & $\mathrm{C}_{2}$ & $\mathbf{M}$ & $\mathrm{C}_{1}$ & $\mathrm{C}_{2}$ & $\mathbf{M}$ & $\mathbf{C}_{1}$ & $\mathrm{C}_{2}$ & $\mathbf{M}$ & $\mathrm{C}_{1}$ & $\mathbf{C}_{2}$ & M & \\
\hline $\mathrm{T}_{1}$ & 18.5 & 18.3 & 18.4 & 19.0 & 17.8 & 18.4 & 15.2 & 13.9 & 14.5 & 15.2 & 14.2 & 14.7 & 8.5 & 8.7 & 8.6 & 14.4 & 8.5 & 11.4 & 14.3 \\
\hline $\mathbf{T}_{2}$ & 18.1 & 17.7 & 17.9 & 18.0 & 18.5 & 18.2 & 18.7 & 15.7 & 17.2 & 17.9 & 16.3 & 17.1 & 9.2 & 9.4 & 9.3 & 15.3 & 8.8 & 12.0 & 15.3 \\
\hline $\mathbf{T}_{3}$ & 18.6 & 18.6 & 18.6 & 18.3 & 18.5 & 18.4 & 18.0 & 15.8 & 16.9 & 16.8 & 15.1 & 15.9 & 9.2 & 9.5 & 9.3 & 15.4 & 8.5 & 11.9 & 15.2 \\
\hline $\mathbf{T}_{4}$ & 19.3 & 19.6 & 19.5 & 18.5 & 18.7 & 18.6 & 17.3 & 15.4 & 16.3 & 17.0 & 16.0 & 16.5 & 12.8 & 9.6 & 11.2 & 15.9 & 9.2 & 12.5 & 15.8 \\
\hline $\mathbf{T}_{5}$ & 19.4 & 20.1 & 19.7 & 18.0 & 18.8 & 18.4 & 18.3 & 17.2 & 17.7 & 18.1 & 17.1 & 17.6 & 14.6 & 16.1 & 15.3 & 17.3 & 12.5 & 14.9 & 17.3 \\
\hline $\mathrm{T}_{6}$ & 17.8 & 18.0 & 17.9 & 18.1 & 18.8 & 18.4 & 16.2 & 16.6 & 16.4 & 16.1 & 16.8 & 16.5 & 8.9 & 9.2 & 9.0 & 15.2 & 8.7 & 11.9 & 15.0 \\
\hline$T_{7}$ & 19.2 & 19.5 & 19.3 & 18.1 & 18.1 & 18.1 & 16.9 & 15.1 & 16.0 & 16.2 & 15.3 & 15.8 & 9.2 & 9.5 & 9.4 & 15.3 & 9.1 & 12.2 & 15.1 \\
\hline$T_{8}$ & 18.3 & 18.5 & 18.4 & 17.8 & 18.3 & 18.0 & 14.8 & 17.3 & 16.0 & 15.4 & 16.0 & 15.7 & 15.4 & 16.5 & 16.0 & 16.3 & 10.6 & 13.4 & 16.2 \\
\hline Mean & 18.6 & 18.7 & 18.7 & 18.2 & 18.4 & 18.3 & 16.9 & 15.8 & 16.4 & 16.6 & 15.8 & 16.2 & 11.0 & 11.0 & 11.0 & 15.6 & 9.5 & 12.5 & 15.5 \\
\hline & & $\mathrm{P}$ & $\mathrm{C}$ & $\mathrm{T}$ & PC & $\mathrm{CT}$ & PT & PCT & & & & & & & & & & & \\
\hline SEd & & 0.02 & 0.01 & 0.02 & 0.03 & 0.04 & 0.07 & 0.1 & & & & & & & & & & & \\
\hline $\mathrm{CD}(\mathrm{P}$ & $0.05)$ & 0.04 & 0.02 & 0.05 & 0.07 & 0.08 & 0.14 & 0.2 & & & & & & & & & & & \\
\hline
\end{tabular}

(MAS - Months after storage; M - Mean; P - Storage period; C - Container; T - Treatment) 
Table.5 Effect of seed treatment and storage container on vigour index in rice variety ADT (R) 46 (pooled data)

\begin{tabular}{|c|c|c|c|c|c|c|c|c|c|c|c|c|c|c|c|c|c|c|c|}
\hline \multirow{2}{*}{$\begin{array}{l}\text { Treat } \\
\text { ments }\end{array}$} & \multicolumn{3}{|c|}{ Initial } & \multicolumn{3}{|c|}{2 MAS } & \multicolumn{3}{|c|}{4 MAS } & \multicolumn{3}{|c|}{6 MAS } & \multicolumn{3}{|c|}{8 MAS } & \multicolumn{3}{|c|}{10 MAS } & \multirow[t]{2}{*}{ Mean } \\
\hline & $\mathrm{C}_{1}$ & $\mathrm{C}_{2}$ & $\mathbf{M}$ & $\mathrm{C}_{1}$ & $\mathrm{C}_{2}$ & $\mathbf{M}$ & $\mathrm{C}_{1}$ & $\mathrm{C}_{2}$ & $\mathbf{M}$ & $\mathrm{C}_{1}$ & $\mathrm{C}_{2}$ & $\mathbf{M}$ & $\mathrm{C}_{1}$ & $\mathrm{C}_{2}$ & $\mathbf{M}$ & $\mathrm{C}_{1}$ & $\mathrm{C}_{2}$ & $\mathbf{M}$ & \\
\hline$T_{1}$ & 3461 & 3379 & 3420 & 3117 & 3171 & 3144 & 2727 & 2457 & 2592 & 2465 & 2359 & 2412 & 1320 & 1428 & 1374 & 1074 & 1167 & 1121 & 2344 \\
\hline $\mathbf{T}_{2}$ & 3393 & 3467 & 3430 & 3426 & 3327 & 3286 & 3197 & 2835 & 3016 & 2832 & 2633 & 2732 & 1568 & 1692 & 1630 & 1128 & 1304 & 1216 & 2552 \\
\hline$T_{3}$ & 3417 & 3487 & 3452 & 3271 & 3366 & 3318 & 3067 & 2909 & 2988 & 2617 & 2656 & 2636 & 1492 & 1617 & 1554 & 1215 & 1249 & 1232 & 2530 \\
\hline $\mathbf{T}_{4}$ & 3550 & 3610 & 3580 & 3199 & 3237 & 3218 & 2908 & 2932 & 2920 & 2691 & 2483 & 2587 & 1707 & 1757 & 1732 & 1332 & 1431 & 1381 & 2569 \\
\hline$T_{5}$ & 3679 & 3755 & 3717 & 3343 & 3490 & 3416 & 3376 & 3407 & 3391 & 3180 & 3263 & 3221 & 1601 & 1819 & 1710 & 1277 & 1401 & 1339 & 2799 \\
\hline $\mathrm{T}_{6}$ & 3327 & 3416 & 3371 & 3093 & 3115 & 3104 & 2860 & 2822 & 2841 & 2484 & 2502 & 2493 & 1433 & 1540 & 1486 & 1175 & 1245 & 1210 & 2417 \\
\hline $\mathbf{T}_{7}$ & 3563 & 3613 & 3588 & 3201 & 3281 & 3241 & 2987 & 3071 & 3029 & 2777 & 2805 & 2791 & 1582 & 1705 & 1643 & 1238 & 1324 & 1281 & 2595 \\
\hline$\overline{T_{8}}$ & 3368 & 3520 & 3444 & 3297 & 3368 & 3333 & 2751 & 2833 & 2792 & 2449 & 2838 & 2643 & 1656 & 1769 & 1712 & 1242 & 1263 & 1252 & 2529 \\
\hline Mean & 3470 & 3531 & 3500 & 3221 & 3294 & 3257 & 2984 & 2908 & 2946 & 2687 & 2692 & 2689 & 1545 & 1666 & 1605 & 1210 & 1298 & 1254 & 2542 \\
\hline & & $\mathrm{P}$ & $\mathrm{C}$ & $\mathrm{T}$ & PC & CT & PT & PCT & & & & & & & & & & & \\
\hline SEd & & 0.1 & 0.08 & 0.1 & 0.2 & 0.2 & 0.4 & 0.5 & & & & & & & & & & & \\
\hline $\mathrm{CD}(\mathrm{P}=$ & .05) & 0.2 & 0.15 & 0.3 & 0.4 & 0.4 & 0.8 & 1.1 & & & & & & & & & & & \\
\hline
\end{tabular}

(MAS - Months after storage; M - Mean; P - Storage period; C - Container; T - Treatment) 
Table.6 Weather data during seed storage period

\begin{tabular}{|c|c|c|c|c|c|c|c|c|}
\hline \multirow{3}{*}{$\begin{array}{c}\begin{array}{c}\text { Year/ } \\
\text { Months }\end{array} \\
\text { January } \\
\end{array}$} & \multicolumn{4}{|c|}{2016} & \multicolumn{4}{|c|}{2017} \\
\hline & \multicolumn{2}{|c|}{$\begin{array}{c}\text { Temp. } \\
\left({ }^{0} \mathrm{C}\right)\end{array}$} & \multirow{2}{*}{$\begin{array}{c}\text { RH } \\
\left(\begin{array}{c}\text { \% } \\
9\end{array}\right.\end{array}$} & \multirow{2}{*}{$\begin{array}{c}\mathbf{R F} \\
(\mathbf{m m}) \\
0\end{array}$} & \multicolumn{2}{|c|}{$\begin{array}{c}\text { Temp. } \\
\left({ }^{0} \mathrm{C}\right)\end{array}$} & \multirow{2}{*}{$\begin{array}{c}\text { RH } \\
(\%) \\
95\end{array}$} & \multirow{2}{*}{ 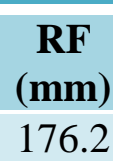 } \\
\hline & 30.7 & 20.4 & & & 29.7 & 19.8 & & \\
\hline February & 33.4 & 21.3 & 95 & 0 & 31.4 & 18.3 & 95 & 0.0 \\
\hline March & 35.3 & 22.9 & 93 & 0 & 34.0 & 23.2 & 95 & 27.2 \\
\hline April & 38.0 & 26.3 & 89 & 0 & 37.9 & 26.0 & 89 & 0.0 \\
\hline May & 36.4 & 26.0 & 87 & 113.8 & 37.3 & 26.5 & 81 & 4.8 \\
\hline June & 34.6 & 25.2 & 87 & 51.2 & 36.1 & 25.4 & 85 & 115.6 \\
\hline July & 34.8 & 25.3 & 80 & 12.9 & 35.9 & 25.6 & 83 & 75.6 \\
\hline August & 35.4 & 25.3 & 78 & 118.4 & 34.4 & 24.9 & 89 & 122.8 \\
\hline September & 34.7 & 25.1 & 81 & 38.8 & 33.7 & 24.4 & 89 & 215.6 \\
\hline October & 35.4 & 24.3 & 86 & 30.8 & 33.0 & 24.6 & 91 & 134.6 \\
\hline November & 31.1 & 22.3 & 92 & 86.8 & 29.5 & 23.5 & 96 & 452.2 \\
\hline December & 29.5 & 20.5 & 93 & 77.7 & 29.3 & 21.6 & 95 & 163.8 \\
\hline Mean & 34.1 & 23.7 & 88.0 & 44.2 & 33.5 & 23.6 & 90.2 & 124.0 \\
\hline
\end{tabular}

The seedling vigour also showed similar trend where, it was maintained maximum upto six months and thereafter drastic decline was noticed in all the treatments. In which, the shoot length was decreased from $17.5 \mathrm{~cm}$ to $9.1 \mathrm{~cm}$ during 10 months storage.

However, the lengthiest shoot $(11.9 \mathrm{~cm})$ was measured in the seeds fortified with $\alpha$ tocopherol @ $1 \%$ (Table 3). Additionally, reduction in root length was recorded from 18.7 $\mathrm{cm}$ to $9.5 \mathrm{~cm}$ at 10 months storage. Nevertheless, the seed fortification with $\alpha$ tocopherol@1\% for $18 \mathrm{~h}$ have maintained better root length $(14.9 \mathrm{~cm})$ compared to control $(11.4 \mathrm{~cm})($ Table 4$)$. Similarly, decline in vigour index (3500 to 1254) was noticed irrespective of the treatments and higher vigour index (2799) was recorded in $\alpha$ - tocopherol treated seed (Table 5).

Therefore, it is concluded that rice variety ADT (R) 46 seeds soaked in $\alpha$-tocopherol @ $1 \%$ for $18 \mathrm{~h}$ and stored in polylined gunny bag have recorded higher germination and seedling vigour upto six months compared to control. Thereafter, seed germination and seedling vigour were decreased drastically irrespective of the treatments.

\section{References}

Abdul-Baki, A.A. and J.D. Anderson. 1973. Vigour determination in soybean seeds by multiple criteria. Crop Sci., 13: 630-633.

Akter, M. A. and I. Hossain. 2015. Quality of some hybrid seeds of rice and control of seed borne fungi in Bangladesh. $J$. Bangladesh Agril. Univ., 13(2): 161 168.

Akter, M.A., A.K.M.K. Hasan, S.A. Uddin and I. Hossain. 2015. Seed treatment for improving quality of hybrid seeds of rice. Asian J. Medic. Bio. Res., 1 (3): 406-415.

Anil Sebastian and P. Selvaraju. 2017. Influence of temperature of production environment on seed quality and storability of rice genotypes. Int. J. Curr. Microbiol. App. Sci., 6(12): 4273-4284.

Basra, S.M.A., M. Farooq, A. Wahid and M.B. Khan. 2006. Rice seed invigoration by hormonal and vitamin priming. Seed Sci. \& Technol., 34: 753-758. 
Begum, A. J. M., B. Venudevan and M. Jayanthi. 2013. Storage fungi in groundnut and the associate seed quality deterioration- A review. Plant Pathol. J., 12(3): 127-134.

Biradar Patil, N.K. and M. Shekhargouda. 2007. Seed storage studies in rice hybrid. Karnataka J. Agric. Sci., 20(3): 618-621.

Caldwell, C.R., S.J. Britz and R.M. Mirecki. 2005. Effect of temperature, elevated carbon dioxide, and drought during seed development on the isoflavone content of dwarf soybean [Glycine max (L.) Merrill] grown in controlled environments. $J$. Agrl. Food Chem., 53 (4): 1125-1129.

Draganic, I. and S. Lekic. 2012. Seed priming with antioxidants improves sunflower seed germination and seedling growth under unfavorable germination conditions. Turk. J. Agric. For., 36: 421428.

ISTA. 1999. International Rules for Seed Testing. Seed Sci. \& Technol., 27: 30- 35.

Jyoti. 2017. Effect of treatment, packing material and storage on viability in paddy (Oryza sativa L.). J. Pharmaco. Phytochem., 6(4): 962-964.

Kad, V.P., Y.P. Khandetod, C.A. Nimbalkar and M.S. Jadhav. 2016. Design and development of paddy storage structure for Konkan region. Int. J. Res. Eng. Technol., 4(5): 25-34.

Kameswara Rao, N. and M. T. Jackson. 1996. Seed longevity of rice cultivars and strategies for their conservation in genebanks. Ann. Bot., 77: 251-260.

Khalid, N., S.A. Anwar, M.I. Hoque, A. Riaz and M.S.A. Khan. 2001. Seed-borne fungi and bacteria of rice and their impact on seed germination. Pakistan J. Phytopathol., 13: 75-81.
Marques, E. R., E.F. Araujo, R.F. Araujo, S.M Filho and P.C. Soares. 2014. Seed quality of rice cultivars stored in different environments. J. Seed Sci., 36(1): 32-39.

Paderes, D.E., T.W. Mew and L.L. Ilag. 1997. Influence of moisture content and length of storage on fungal invasion of paddy rice. Biotropia, 10: 1-13.

Pameri, M.S., A.K. Chaurasia and A.W. Hekmat. 2016. Influence of packaging materials on germination and seed vigour characters of rice (Oryza sativa L.) during storage. Int. J. Multi. Res. Develop., 3(7): 317-320.

Panse, V. G. and P.V. Sukhatme. 1967. Statistical Method for Agricultural Workers, ICAR Publication, New Delhi.

Purushattam, S.P., K.L. Patkar, H.S. Prakash and H.S. Shetty. 1996. Storage fungi and their influence on rice seed quality. Indian Phytopathol, 49: 152-156.

Sultana, N., Y. Ali, S. Jahan and S. Yasmin. 2016. Effect of storage duration and storage devices on seed quality of Boro Rice variety BRRI Dhan47. J. Plant Pathol. Microbiol., 8: 392.

Toledo, M.Z., N.R. Fonseca, M.L. Cesar, R.P. Soratto, C. Cavariani and C.A.C. Crusciol. 2009. Qualidade fisiológica earmazenamento de sementes de feijão em função da aplicação tardia de nitrogênio em cobertura. Pesquisa Agropecuária Tropical, 39 (2): 124-133.

Yogalakshmi, J., A.S. Ponnuswamy and T.V. Karivaratharaju. 1996. Seed storage potential of rice hybrid (CORH-1) and parental lines. Madras Agric. J., 83: 729732.

\section{How to cite this article:}

Raja, K. and Sasikala, K. 2018. Effect of Seed Treatments and Storage Containers on Viability and Vigour of Rice (Oryza sativa L.) Variety ADT (R) 46 Seeds. Int.J.Curr.Microbiol.App.Sci. 7(09): 3087-3096. doi: https://doi.org/10.20546/ijcmas.2018.709.385 